\title{
Udo Steiner zum 70. Geburtstag
}

Am 16.9. 2009 vollendete Udo Steiner das 70. Lebensjahr. Geboren in Bayreuth studierte er Rechtswissenschaften in Erlangen, Saarbrücken und Köln. In Erlangen war er zunächst Wissenschaftliche Hilfskraft, später Wissenschaftlicher Assistent von Klaus Obermayer am Institut für Kirchenrecht und Öffentliches Recht. Nach Promotion (1965) und Habilitation (1972) führte ihn sein akademischer Werdegang an die Fakultät für Rechtswissenschaften der Universität Bielefeld, wo er zunächst zum Wissenschaftlichen Rat und Professor, später zum ordentlichen Professor ernannt wurde. 1979 folgte er einem Ruf auf die Nachfolge von Franz Mayer auf einen Lehrstuhl an der Juristischen Fakultät der Universität Regensburg, der er - trotz eines für ihn sehr reizvollen Rufes an die Deutsche Hochschule für Verwaltungswissenschaften Speyer im Jahr 1985 - treu blieb. Von 1995 bis 2008 war er Richter des Bundesverfassungsgerichts (1. Senat).

Die erste wissenschaftliche Veröffentlichung Steiners ist seine mit dem Fakultätspreis ausgezeichnete Dissertation „Verfassunggebung und verfassunggebende Gewalt des Volkes“. In ihr wendet sich Steiner vor allem gegen die Vorstellung einer prinzipiell unterschiedlichen Qualität von verfassungändernder und verfassunggebender Gewalt. Auch die Verfassunggebung sei als rechtlich gebundener, keinesfalls als ungebundener revolutionärer Prozess zu verstehen. Das Grundgesetz als Verfassungsordnung bestimme auch das Verfahren und den Umfang der künftigen Verfassungserzeugung in verbindlicher Weise. Auf das Thema Verfassunggebung und Verfassungänderung kam Steiner in der Folge allerdings nur noch vereinzelt zurück. Sehr viel stärker geprägt ist sein wissenschaftliches Gesamtwerk von seiner Habilitationsschrift „Öffentliche Verwaltung durch Private“. Bis heute kontrovers diskutiert wird das darin entwickelte Verständnis von Beleihung im Sinne der Übertragung staatlicher Aufgaben auf Private, im Gegensatz zur immer noch herrschenden, aber oft sehr undifferenziert und ungenau formulierenden herrschenden Meinung, die das Wesen von Beleihung in der Übertragung von Befugnissen sehen will. In der Folgezeit immer wieder als richtig erwiesen hat sich die weitere These Steiners, dass der Staat das Institut der Beleihung vor allem einsetzt, um den besonderen Sachverstand Privater bei der Erfüllung öffentlicher Aufgaben zu nutzen. Die Figur des 
Beliehenen ist in der modernen Gesetzgebung sehr viel präsenter, als dies gemeinhin vermutet wird.

Das grundsätzliche Verfassungsverständnis Steiners zeigt vor allem der Vortrag auf der Staatsrechtslehrertagung 1984 in Köln zum Thema „Kulturauftrag im staatlichen Gemeinwesen “. Steiner plädiert (auch in seinen daran anschließenden weiteren Veröffentlichungen zu diesem Thema) für die Wahrung des Grundgesetzes als „Juristenverfassung“ und gegen den Weg in eine „Pastorenverfassung“. Er wendet sich gegen die permanente Findung oder Erfindung von „Teilverfassungen“ (etwa einer Kulturverfassung), gegen die unbesehene Gleichstellung einer Freiheitsgarantie für die Kunst (Art.5 Abs. 3 GG) mit einer Art Kulturstaatsgarantie, gegen die Vorstellung, Staat und Kommunen seien institutionell in Sachen der Kunstförderung zur Neutralität zu verpflichten und auch gegen die Ableitung finanzieller Förderverpflichtungen des Staates etwa für Opern und Theater. Vorschläge, die Verfassung mit weiteren Staatszielbestimmungen (etwa einer „Kulturstaatsklausel“) „anzureichern“, verweist er (in der Regel sogar bei seinem Lieblingsthema „Sport“) an die Landesverfassunggeber.

Das wissenschaftliche Interesse Steiners war stets breit angelegt. Seine Veröffentlichungen reichen von staatsrechtlichen Grundfragen etwa zur Religionsfreiheit bis hin zu Spezialfragen des Besonderen Verwaltungsrechts. So wird man davon ausgehen können, dass er der einzige deutsche Staatsrechtslehrer ist, den das Thema der „Körung“ zu rechtswissenschaftlichen Forschungen animiert hat. Viele in Fachzeitschriften erschiene Beiträge beruhen auf Vorträgen, für die Steiner bis heute extrem nachgefragt wird. Besondere mediale Aufmerksamkeit erlangte er bei der Vorbereitung des sog. Zweiten Abtreibungsurteils des Bundesverfassungsgerichts (BVerfGE 88, $203 \mathrm{ff}$.), an der er als Prozessvertreter des Freistaates Bayern federführend mitwirkte.

Zwei „Lieblingsgebiete“ kristallisieren sich im wissenschaftlichen Wirken von Udo Steiner jedoch klar heraus: das Straßen- und Straßenverkehrsrecht mit seinen Bezügen zum Planungs- und Kommunalrecht einerseits und das Sportrecht andererseits. Mit diesen beiden Interessensschwerpunkten korrespondieren bis heute vielfältige ehrenamtliche Funktionen (ohne Anspruch auf Vollständigkeit): Mitglied des Forschungsausschusses Straßenrecht der Forschungsgesellschaft für Straßenwesen (ab 1970), Berufung in den Beirat für Grundsatzfragen beim Vorstand der Deutschen Bundesbahn (ab 1986), Berufung in den Wissenschaftlichen Beirat des Deutschen Sportbundes und in den Beirat des Konstanzer Arbeitskreises für Sportrecht (1986), Berufung in den Beirat für Grundsatzfragen des Deutschen Sportbundes (1991). Er gehört dem Herausgeberkreis der Zeitschrift für Sport und Recht (SpuRt, seit 1994), der Zeitschrift Deutsches Autorecht (DAR, seit 1995) und der bei Duncker und Humblot erscheinenden Reihe „Beiträge zum Sportrecht“ an. Der Aufschwung des Sportrechts von einer zunächst von manchen belächel- 
ten Randerscheinung zu einem anerkannten Rechtsgebiet ist untrennbar mit seinem Namen verbunden.

Als Mitglied des 1. Senates des Bundesverfassungsgerichts war Steiner vor allem für das von Richtervorlagen nach Art. 100 Abs. 1 GG dominierte und dadurch besonders arbeitsintensive Sozialrecht zuständig. 40 verfahrensabschließende Entscheidungen wurden in diesem Bereich von Steiner vorbereitet, der thematische Bogen reicht von der sog. Stasi-Rente (BVerfGE 100, 138 ff.) bis zur Kostenerstattung bei künstlicher Befruchtung (BVerfGE 117, 316 ff.). Vor allem ihm ist die verfassungsrechtliche Bewältigung der sozialrechtlichen Fragen der Deutschen Wiedervereinigung zu verdanken, die mittlerweile als weitgehend abgeschlossen angesehen werden kann.

Nach seinem Ausscheiden aus dem Amt des Richters des Bundesverfassungsgerichts und der gleichzeitigen Emeritierung in Regensburg hat Steiner weitere Funktionen übernommen. Seit 2008 fungiert er als Ombudsmann der Deutschen Bahn AG, als Mitglied des Deutschen Sportschiedsgerichts, als Vorsitzender der Anti-Dopingkommission des Deutschen Olympischen Sportbundes und des Ständigen Schiedsgerichts des Deutschen Fußballbundes und der Deutschen Fußball Liga.

Steiner war und ist ein glänzender akademischer Lehrer, für seine bisher über 80 Doktoranden und 3 Habilitanden ein ebenso fürsorglicher wie umsichtiger Doktor- und Habilitations, ,vater“. Es ist ein großes Glück für die Regensburger Studierenden, dass er auch nach seiner Emeritierung und trotz vielfältiger anderer Verpflichtungen weiterhin Veranstaltungen anbietet, vor allem Seminare zum Verfassungs- und Verwaltungsrecht. Für seine vielfältigen Aufgaben ist ihm für die Zukunft vor allem eine weiterhin gute Gesundheit zu wünschen. Es ist derzeit nicht erkennbar, wie der deutsche Sport seine vielfältigen Probleme nicht nur bei der Doping-Bekämpfung ohne seinen fachlichen Rat bewältigen könnte. Aber auch viele andere freuen sich auf weitere gemeinsame Projekte, Gespräche und Begegnungen.

Gerrit Manssen 